\title{
ENVOLVIMENTO DO CONSUMIDOR NO PROCESSO DE DESENVOLVIMENTO DE PRODUTOS COMO MEDIDA DE SUCESSO: UM ESTUDO COM EMPRESAS PARTICIPANTES DOS PRÊMIOS FINEP E NACIONAL DE INOVAÇÃO
}

\author{
Ana Paula Matias \\ Mestre em Administração pela Universidade do Vale do Itajaí - UNIVALI \\ Professora da Faculdade Capivari - FUCAP \\ anamatias@fucap.edu.br (Brasil)
}

\section{Ricardo Boeing da Silveira}

Doutor em Administração de Empresas pela Fundação Getulio Vargas - FGV

Professor da Universidade do Vale do Itajaí - UNIVALI

boeing@univali.br (Brasil)

\section{Marcelo Moll Brandão}

Doutor em Administração de Empresas pela Fundação Getulio Vargas - FGV

Professor da Programa de Pós-gradução da Universidade Nove de Julho - UNINOVE mollmkt@gmail.com (Brasil)

\section{RESUMO}

Este estudo teve como objetivo analisar os níveis de envolvimento dos consumidores em processos de PDP (Processo de Desenvolvimento de Produtos), como medida de sucesso no lançamento de novos produtos, além de discutir, a partir dos resultados que foram encontrados, se os prêmios de inovação no Brasil, são realmente para premiar produtos e/ou projetos inovadores, ou se são apenas prêmios de promoção da inovação. Para alcançar os objetivos a que se propôs este trabalho, optou-se pela abordagem quantitativa do tipo survey e descritiva quanto aos seus fins. A coleta de dados foi realizada através de questionário eletrônico, encaminhado via e-mail aos gestores das empresas classificadas e vencedoras nos prêmios FINEP e Nacional de Inovação, entre os anos de 2008 e 2011. Para as análises estatísticas multivariadas dos dados utilizou-se o teste não paramétrico Mann-Whitney, a análise discriminante e a regressão linear com variável Dummy, as análises estatísticas univariadas utilizam a análise de variância - ANOVA. A tabulação dos dados foi feita utilizando o software SPSS (Statistical Package for the Social Sciences). Os resultados obtidos demonstram que, $100 \%$ das empresas estudadas possuem algum tipo de mecanismo de envolvimento com o consumidor implantado. Constatou-se também, que as ferramentas de interação com o consumidor menos utilizado por essas empresas são as virtuais.

Palavras chave: Prêmios de inovação; Envolvimento com o consumidor; PDP. 


\section{INTRODUÇÃO}

$\mathrm{Na}$ atual conjectura do mercado, principalmente devido à globalização, os produtos brasileiros têm que lutar diariamente para manter a competitividade, em preço e qualidade, em relação aos similares internacionais, tanto os advindos de países mais desenvolvidos, com elevado aparato tecnológicos, quanto os vindos de países em que os custos de fabricação estão muito abaixo dos brasileiros.

Vargo e Lusch (2004) ressaltam que quando se trata de envolvimento do consumidor em PDP (Processo de Desenvolvimento de Produtos), a participação dos clientes não acaba na linha de produção, este é um processo "meio" e não "fim”. Mesmo depois que o cliente compra o produto, ele ainda precisará aprender a usar, adaptar e consertar às suas necessidades, ou seja, em resumo, o processo envolve desde a criação de um produto até os serviços de pós-venda envolvidos na plena satisfação do consumidor. Griffin (1997) diz que um processo bem definido é um dos principais fatores de sucesso no desenvolvimento de produtos.

Lançar novos produtos tem sido a forma utilizada pelas organizações para obter vantagem em relação aos concorrentes, por isso é de grande importância que se utilize mecanismos e metodologias que contribuam para o sucesso desses novos produtos depois de lançados (SLATER e NARVER, 1995; PRAHALAD e RAMASWANY, 2004; SAWHNEY et al, 2005; JUGEND et al, 2006; BROWN e EISENHARDT, 1995).

Empresas que desejam ter comportamentos inovadores no mercado precisam buscar alternativas complementares as técnicas de pesquisa de mercado. (SLATER e NARVER, 1995). Ambientes globais, intensos e dinâmicos, requerem que o PDP torne-se foco de competição. Prahalad e Ramaswany (2004, p.3) mencionam que "interações de alta qualidade que permitem ao cliente cocriar experiências únicas com a empresa são a chave para desvendar novas fontes de sucesso". As interações entre cliente/empresa quando não bem conduzidas podem trazer efeitos negativos, por outro lado se bem implantadas podem se tornar um grande diferencial para a organização (NAMBISAM, 2002).

Nesse contexto, este trabalho pretende oferecer contribuições teórico-empíricas tanto para a academia quanto para o mercado que justifiquem o envolvimento do consumidor nos processos de PDP, além de discutir, a partir dos resultados que forem encontrados, se os prêmios de inovação no Brasil, são realmente para premiar produtos e/ou projetos inovadores, ou se são apenas prêmios de promoção da inovação; e se as inovações geradas no Brasil são apenas incrementais e por isso não

Revista de Administração e Inovação, São Paulo, v.12, n.1, p.174-200, jan./mar. 2015. 
Envolvimento do consumidor no processo de desenvolvimento de produtos como medida de sucesso: Um estudo com empresas participantes dos prêmios Finep e Nacional de Inovação

geram vantagem competitiva. Este envolvimento torna-se vantajoso tanto para a organização, que dispõe através da cocriação de valor, das informações necessárias para oferecer aos clientes produtos exatamente no formato desejado, permitindo-lhes a tão desejada vantagem competitiva, quanto para o cliente que recebe produtos que melhor supram suas necessidades.

Percebe-se que os investimentos em PDP não são em vão, principalmente quando esses investimentos são direcionados para que o consumidor tenha participação no processo, melhorando as chances de sucesso pela diminuição das diferenças entre o que a empresa acredita ser o melhor e o que o consumidor considera ideal, sendo assim, este artigo objetiva contribuir para a referida construção teórica na área e suprir a lacuna deixada no estudo de Santos e Brasil (2010), que dizem que futuros estudos podem ser realizados para constatar-se se maiores níveis de envolvimento resultam em sucesso no lançamento de novos produtos?

Sendo assim, a presente pesquisa é desenvolvida a partir da seguinte questão: Quais os níveis de envolvimento dos consumidores em processos de PDP, como medida de sucesso no lançamento de novos produtos?

Embora já existam alguns estudos sobre PDP, o tema ainda continua a ser uma "caixa preta" a ser explorada, e essencial para o desenvolvimento da inovação (BROWN e EISENHARDT, 1995). A cocriação de valor continua a ser um tema relevante para pesquisas, pois é preciso integrar passado e futuro para alcançar maiores níveis de crescimento organizacional (CHANDLER e VARGO, 2011). Não obstante as significâncias do tema ainda existem poucos estudos sobre os níveis de envolvimento do consumidor em processos de PDP e prêmios de inovação como medida de sucesso (SANTOS e BRASIL, 2010).

O objetivo desta pesquisa é analisar os níveis de envolvimento dos consumidores em processos de PDP, como medida de sucesso no lançamento de novos produtos. Para o alcance deste objetivo traçaram-se alguns objetivos específicos, sendo eles: Destacar, na percepção dos gerentes, os diferentes níveis de envolvimento dos consumidores em processo de PDP; Avaliar os mecanismos de interação com os consumidores, utilizados pelas empresas estudadas; Identificar o sucesso de novos produtos lançados após os diferentes níveis de envolvimento dos consumidores em processos de PDP; e Comparar o PDP, a Inovação e o Sucesso entre Empresas Vencedoras e Classificadas nos Prêmios FINEP e Nacional de Inovação. Com a finalidade de atender os objetivos deste estudo, optou-se por realizar uma pesquisa descritiva, de abordagem quantitativa e estratégia do tipo survey.

Revista de Administração e Inovação, São Paulo, v.12, n.1, p.174-200, jan./mar. 2015. 
Para melhor entendimento deste artigo, ele foi dividido em seções, sendo elas: Introdução, Referencial Teórico, Metodologia de Pesquisa, Análises e Resultados da Pesquisa, Conclusão e Referências.

\section{REFERENCIAL TEÓRICO}

\subsection{Estratégias em PDP e suas Tendências}

Quando aplicado ao contexto empresarial o conceito de estratégia abrange todas as táticas e técnicas escolhidas em âmbito corporativo em busca de cumprir os planos e alcançar os objetivos institucionais. Skinner (1969) diz que estratégia é um conjunto de planos e políticas pelos quais uma empresa objetiva ganhar vantagem competitiva sobre seus concorrentes.

O desenvolvimento de produto é fundamental para a viabilidade das empresas, mas ainda é um desafio à compressão dos resultados das pesquisas relacionadas porque a literatura do tema é muito fragmentada e variada (BROWN e EISENHARDT, 1995). Percebe-se, porem, que as empresas que estão se apropriando dos conceitos de vantagem competitiva e entendo a importância do PDP dentro das organizações, são as que estão conquistando um forte espaço no mercado.

Dentre as estratégias utilizadas pelas organizações a cocriação através do envolvimento dos clientes em processos de desenvolvimento de produtos (PDP), tem se mostrado bastante produtiva (SHOCKER e SRINIVASAN, 1979).

Pode-se perceber que as empresas que exploram melhor o envolvimento do consumidor em seus processos de desenvolvimento de produtos obtém mais sucesso, pois conseguem identificar o que o cliente realmente gosta ou não no produto, antes mesmo de seu lançamento.

Uma boa tomada de decisão, no que tange ao envolvimento do cliente em PDP, logo no início do processo pode melhorar os resultados e gerar vantagem competitiva para a empresa (ROZENFELD et al. 2006; JUGEND et al. 2006), sendo assim, este artigo procura entender se há relação significativa entre o envolvimento do consumidor em PDP e o sucesso de novos produtos.

O fato de o cliente estar presente no processo de cocriação de valor permite que a empresa tenha mais sucesso no lançamento de seus produtos, a questão principal é se esses prêmios de inovação no Brasil buscam premiar ações inovadoras ou simplesmente promover a inovação.

No atual cenário econômico, marcado por grande competitividade, qualidade dos produtos e concorrência acirrada, o sucesso organizacional depende da capacidade de inovação tecnológica da

Revista de Administração e Inovação, São Paulo, v.12, n.1, p.174-200, jan./mar. 2015. 
empresa, colocando novos produtos no mercado, com custo-benefício maior para o cliente, qualidade melhor e em velocidade maior do que a de seus concorrentes (SBRAGIA e ANDREASSI, 2002).

Percebe-se que o envolvimento do consumidor em PDP, pode resultar em menor tempo para o lançamento de novos produtos e inovações, gerando o diferencial competitivo almejado pelas organizações. Segundo Garcia (2008) os prêmios de inovação, são muito utilizados em vários países e em segmentos restritos de mercados, fazendo parte de avaliações individuais das empresas e de seu caráter inovador.

\subsection{Cocriação de Valor}

Von Hippel (1988) descreve a importância da interação com clientes no desenvolvimento de novos produtos. Apesar de as empresas é que são vistas como inovadoras e não os clientes, este fato se deve ao poder que a empresa tem para divulgar suas inovações.

Percebe-se que o que ocorre é que o cliente tem ideias inovadoras, que podem fazer grande diferença no mercado, e as empresas aproveitam da interação para desenvolver, melhorar e transformar em produto ou serviço a ideia original.

A cocriação de valor pressupõe que o consumidor esteja presente no momento da criação de novos produtos e/ou aperfeiçoamento de produtos existentes, partilhando com a empresa ideias de inovação, design ou novos produtos (VARGO e LUSCH, 2004). Apesar de Vargo e Lusch (2004), apresentarem diferenças sutis entre cocriação de valor e coprodução de valor (Chathoth et. al, (2013) cita que a coprodução é centrada na empresa, enquanto a cocriação é centrada no consumidor e em experiências) a maioria dos autores trata estes termos como sinônimos. Sendo assim, para este estudo, os termos assim serão considerados.

Fica claro que o cliente não deve apenas ser envolvido no processo de sugestões de melhorias ou de novos produtos e serviços, ele deve também contar às experiências que teve, como se sentiu, o que lhe frustrou, enfim, as empresas devem saber se o cliente considera que produto/serviço vendido cumpriu efetivamente o que era esperado e se o sentimento despertado foi de atendimento e/ou superação das expectativas.

Há formas de interação entre organizações e clientes, entre as mais tradicionais podem-se citar as entrevistas em profundidade, as visitas e reuniões dos consumidores com a equipe de pesquisa e desenvolvimento de produtos, o brainstorming com consumidores, a observação consumidores, o 
recebimento de telefonemas, fax, e-mail e a realização de focus group (ALAM, 2002; EDVARDOSN et. al, 2010).

As empresas já utilizam ambientes virtuais para a cocriação de valor, sendo utilizados fóruns de discussão, e-mail, jogos para customização, design e prototipagem virtual. Em suma, através da combinação de várias tecnologias, as empresas podem fornecer uma gama de serviços online para seus clientes que facilitam diferentes tipos de parcerias em inovação de produtos e criação de valor (DOGSON, 2006; NAMBISAN, 2002; NAMBISAN e BARON 2007; SAWHNEY et al., 2005).

Diante do exposto percebe-se que as empresas passam a contar cada vez mais com seus clientes para descobrir como satisfazê-los, de forma dinâmica, objetiva e interativa. Cabe às organizações saber aproveitar as oportunidades oferecidas, transformando-as em resultados positivos. Para isso, é importante entender todas as partes do processo de desenvolvimento de produtos, bem como suas tendências, pois só dessa forma as organizações alcançarão o sucesso que galgam, em cada um dos índices que podem ser utilizados para medir o sucesso da organização e dos produtos.

\subsection{Medidas de Sucesso}

Venkatraman e Ramanujam (1986), dizem que a medição de sucesso é um dos temas mais difíceis do ambiente de pesquisa. Embora exista um grande volume de literatura sobre o tema não há acordos sobre definições e terminologias.

Alguns autores (BROWN e EISENHARDT, 1995; ERNST, 2002 apud JUGEND 2006) apontam cinco importantes fatores de sucesso ligados às características organizacionais do PDP: a montagem de equipes multifuncionais, autoridade e responsabilidade do líder de projeto, a extensão da responsabilidade sobre o projeto pela equipe de desenvolvimento, o comprometimento de membros da equipe e a intensidade de comunicação durante o projeto.

Avaliar o sucesso de projetos pode ter diferentes significados dependendo do avaliador. Hughes et al. (2004), dizem que a medida do sucesso depende do projeto, ou seja, para cada projeto é preciso construir uma métrica diferente. Em alguns projetos o sucesso é alcançado através de métricas de segurança, em outros em valores financeiros, em outros ainda em termos de desempenho.

Conforme abordado até o momento, são diversos os indicadores de sucesso existentes na literatura, porém, para realização deste trabalho, serão considerados indicadores de vendas, ou seja, serão analisados se os produtos com envolvimento do consumidor no PDP possuem maior índice de recompra que os demais.

Revista de Administração e Inovação, São Paulo, v.12, n.1, p.174-200, jan./mar. 2015. 


\section{METODOLOGIA DE PESQUISA}

Com a finalidade de atender o objetivo deste estudo, optou-se por realizar uma pesquisa descritiva, de abordagem quantitativa e estratégia do tipo survey. A pesquisa quantitativa do tipo survey, foi realizada através da aplicação de questionários com gestores de PDP sobre os tipos de interação existentes entre a empresa e seus clientes.

Para a escolha das empresas a serem pesquisadas, foi considerado como critério o reconhecimento externo por iniciativas de lançamento de produtos. Sendo assim, decidiu-se usar no estudo empresas que foram vencedoras em prêmios de inovação, bem como empresas classificadas nos mesmos prêmios, como critério de comparação. A escolha dos prêmios a serem utilizados se deu ao fato dos mesmos terem reconhecimento nacional, fazendo parte inclusive de outros estudos (SANTOS e BRASIL, 2010; Instituto Inovação, 2005; GARCIA, 2008), a saber: a) Prêmio FINEP de Inovação premiação organizada pela Financiadora de Estudos e Projetos, empresa pública vinculada ao Ministério da Ciência e Tecnologia, que visa premiar os esforços de inovação tecnológica no país; e b) Prêmio Nacional de Inovação - premiação organizada pela Confederação Nacional da Indústria - CNI, premia as empresas que investem em estratégias voltadas para o crescimento do país, estimulando boas práticas, ações inovadoras e de incremento ao segmento industrial.

Foram utilizados dados de 2008 a 2011, pois não há em nenhum dos dois prêmios dados divulgados sobre os vencedores e indicados ao prêmio em 2007, além disso, no caso do Prêmio Nacional de Inovação em sua concepção o mesmo foi criado para premiar empresas com enfoque em qualidade, sendo que até 2005 os critérios para avaliação eram baseados em qualidade, e não em inovação, sendo dessa forma excluídas do objeto de pesquisa.

Com relação ao tamanho da amostra foram pesquisadas 72 empresas, divididas entre classificadas para o Prêmio FINEP de inovação e o Prêmio Nacional de Inovação e vencedoras dos prêmios. Para a identificação da amostra a ser pesquisada, foi realizado contato telefônico e através de e-mail com o Prêmio FINEP de Inovação que retornou com a relação de empresas classificadas e ganhadoras do prêmio. Para a elaboração da amostra referente ao Prêmio Nacional de Inovação, os dados foram retirados da página on-line da CNI, pois não houve retorno das tentativas de contato via email e telefone.

Revista de Administração e Inovação, São Paulo, v.12, n.1, p.174-200, jan./mar. 2015. 
A coleta de dados foi realizada através de dados primários e secundários, sendo que os dados primários foram obtidos através de questionários encaminhados as empresas selecionadas, em que foram previamente encontrados os responsáveis por setores relacionados ao tema de pesquisa. Após esta identificação, utilizaram-se os endereços eletrônicos destes responsáveis para solicitar a participação na pesquisa. Assim, foram encaminhados e-mails, com o link do questionário de pesquisa, pela ferramenta de formulário do Google Docs, além do arquivo em Microsoft Word, visando fornecer uma segunda opção aos respondentes.

Durante o período de setembro a outubro de 2012, fizeram-se contatos telefônicos solicitando a participação na pesquisa, para garantir que o responsável apontado pela empresa respondesse o questionário. Os dados secundários foram obtidos através dos sites do Prêmio FINEP de Inovação e do Prêmio Nacional de Inovação, dos quais foram retiradas a relações de empresas indicadas aos prêmios, bem como as vencedoras.

Entre as análises multivariadas estão o teste não paramétrico Mann-Whitney, para testar as médias dos fatores Inovação, PDP e Sucesso, a análise discriminante e regressão linear com variável Dummy, variável nominal que classifica as empresas em vencedoras (0) e classificadas (1).

Nesta pesquisa foram utilizadas como variáveis independentes Inovação, Cocriação de Valor e Processo de Desenvolvimento de Produtos, e como variável dependente medida de sucesso de novos produtos, isto porque, segundo o objetivo do trabalho, busca-se responder se o maior nível de envolvimento do consumidor em processos de PDP, pode ser considerado medida de sucesso no lançamento de novos produto.

Procurou-se através das características levantadas nos questionários identificar se as empresas vencedoras possuíam características em comum em relação à PDP e Inovação, dentro de seu grupos, que as diferencia-se das características comuns as empresas apenas classificadas. Para a análise dos testes de normalidade usou-se como pressuposto o fato de que a hipótese nula é de que os dados possuem distribuição normal, então nesse caso, não se quer rejeitá-la. As análises estatísticas univariadas utilizam a análise de variância - ANOVA, a qual é utilizada para comparação simultânea das médias.

\section{ANÁLISES E RESULTADOS DA PESQUISA}

Houve retorno de 45 questionários respondidos, sendo desconsiderados cinco, por estarem incompletos, obtendo-se o resultado apresentado a partir da análise de 40 questionários válidos.

Revista de Administração e Inovação, São Paulo, v.12, n.1, p.174-200, jan./mar. 2015. 


\subsection{Destaque dos Níveis de Envolvimento dos Consumidores em PDP}

Nesta seção, destacam-se os níveis de envolvimento dos consumidores em PDP das empresas vencedoras e das empresas classificadas, conforme gráfico apresentado a seguir, com base nos questionários aplicados.

É importante ressaltar que dos 40 questionários respondidos válidos, 12 pertenciam a empresas vencedoras dos prêmios e 28 a empresas classificadas. O gráfico a seguir, mostra a distribuição dos níveis de envolvimento dos consumidores em PDP, entre empresas classificadas e vencedoras dos prêmios FINEP e Nacional de Inovação.

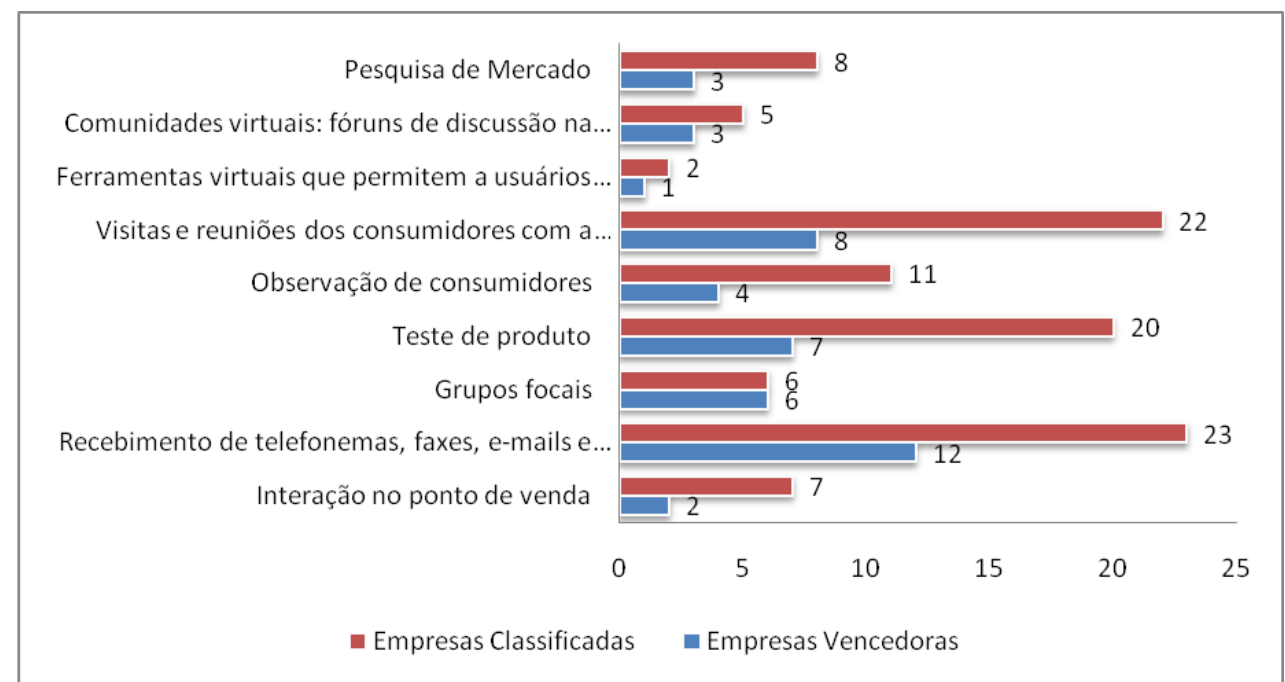

\section{Gráfico 1: Distribuição por mecanismo de interação utilizado classificado entre empresas vencedoras e classificadas. \\ Fonte: Dados da Pesquisa}

Embora a literatura defenda que o envolvimento do consumidor em PDP tornou-se uma técnica alternativa para as organizações coletarem informações de grande valor, em busca de vantagem competitiva por meio do lançamento de produtos inovadores (VON HIPPEL, 1988; ALAM 2002 e NAMBISAN, 2002), o mesmo não se percebe no gráfico anterior, pois mesmo levando-se em conta que há maior número de empresas classificadas do que vencedoras que participaram da pesquisa, não há diferenças significativas entre os mecanismos de interação utilizados por ambos os grupos.

Os resultados da pesquisa apontam que a maioria das empresas utilizam apenas mecanismos de envolvimento com o consumidor de moderada intensidade, ou seja, o consumidor participa do 
processo de desenvolvimento do produto apenas através de técnicas tradicionais, não há grande comunicação bilateral entre empresa e consumidor.

\subsection{Avaliação dos mecanismos de interação com os consumidores utilizados pelas empresas estudadas}

Através dos dados coletados percebe-se que, todas as empresas estudadas possuem mecanismos de interação com os consumidores, algumas de forma mais desenvolvida, outras apenas com mecanismos básicos. Entre as modalidades mais tradicionais de coleta de dados, Alam (2002) encontrou em seu estudo, as seguintes técnicas: entrevistas pessoais em profundidade, visitas e reuniões dos consumidores com a equipe de desenvolvimento de produto, técnica de brainstorming com consumidores, observação de consumidores, recebimento de telefonemas, faxes, email-s e realização de grupos focais.

Percebe-se que apesar de as técnicas de coleta de dados citadas por Alam (2002), serem consideradas as mais tradicionais, as empresas participantes deste estudo não aproveitam todos os benefícios destas técnicas, apesar de Prahalad e Ramaswamy (2004) argumentarem que no futuro, a vantagem competitiva será fortemente relacionada com o processo de cocriação de valor com os consumidores. Esta interação com o consumidor se dá pelo oferecimento das suas habilidades, competências e principalmente pelas suas experiências de consumo. Dessa forma, o consumidor é considerado como um agente ativo e endógeno no processo de marketing (LUSCH, 2007; PRAHALAD e RAMASWAMY, 2004).

O mecanismo denominado como recebimento de telefonemas, faxes, e-mails e cartas com sugestões de novos produtos foi encontrado na maioria das empresas presentes na amostra deste estudo. Ao analisar o conteúdo dos questionários, é possível verificar que a entrada passiva de informações através deste mecanismo é muito utilizada pelas empresas.

As visitas e reuniões dos consumidores com a equipe de PDP podem ser consideradas uma forma mais ativa de envolvimento do consumidor em PDP, através destas visitas e reuniões é possível que a equipe de desenvolvimento de produtos obtenha impressões, desejos e necessidades de seus consumidores. Observou-se nesta pesquisa que 75\% das empresas pesquisadas utilizam esta ferramenta de envolvimento.

Dentre as técnicas de validação, o teste do produto é citado como uma ferramenta que possibilita aos consumidores tirarem suas primeiras impressões sobre o produto que está sendo desenvolvido, através do manuseio de protótipos ou discutindo com representantes da organização ou

Revista de Administração e Inovação, São Paulo, v.12, n.1, p.174-200, jan./mar. 2015. 
com outros consumidores, como resultado da pesquisa, 67,5\% das empresas estudadas disseram utilizar este mecanismo como ferramenta de envolvimento com o consumidor.

Segundo Nambisam e Baron (2007) a interação com os consumidores está sendo desenvolvida em grande parte devido às novas ferramentas tecnológicas disponíveis aos consumidores, algumas delas de baixo custo, fácil utilização e conveniência aos consumidores, porem, o que se percebeu na pesquisa é que dentre as empresas estudadas as ferramentas menos utilizadas são as virtuais, sendo que apenas 7,5\% das empresas apontaram utilizar esta ferramenta, o que demonstra que estas empresas estão deixando de aproveitar grandes oportunidades de interação com seus consumidores.

Percebe-se que as empresas estudadas pouco fazem uso das ferramentas virtuais a sua disposição, perdendo talvez, uma oportunidade valiosa de promover a interação com o consumidor, conhecendo suas preferências e desejos há um custo inferior as técnicas presenciais, isso devido até ao fato de se poder contar com consumidores de diferentes populações demográficas e geográficas em um mesmo ambiente virtual. Estas ferramentas podem ser utilizadas para a promoção de bate-papos, criação de protótipos on-line, preenchimento de pesquisas do tipo survey, entre outros.

Outro mecanismo de envolvimento com o consumidor pouco citado pelas empresas estudadas é a pesquisa de mercado, que segundo Malhotra (2006, p. 32) "é a identificação, coleta, análise e disseminação de informações de forma sistemática e objetiva, e o uso dessas informações para assessorar a gerência na tomada de decisões relacionadas à identificação e solução de problemas (e oportunidades) de marketing”.

Em relação ao mecanismo de observação de consumidores, apenas 37,5\% das empresas disseram utilizar, apesar disso, Kaulio (1998) sugere que a aplicação deste tipo de mecanismo proporciona o desenvolvimento de "design para consumidores", ou seja, o produto é desenvolvido com a observação das necessidades e desejos dos consumidores. Entende-se que as empresas que utilizam este mecanismo conseguem desenvolver seus produtos com grande assertividade, pois o fazem de forma totalmente voltada aos desejos e necessidades dos consumidores.

A utilização de grupos focais também não se destacou entre as empresas estudadas (30\%), porem é definida por Gomes e Barbosa (1999) como um grupo de discussão informal e de tamanho reduzido, com a intenção de obter informações de caráter qualitativo em profundidade. $\quad$ Em relação à interação no ponto de venda, citado por apenas $22,5 \%$ das empresas, corresponde às ações desenvolvidas nos locais de venda dos produtos da organização, através tipo de mecanismo entende-se que o representante da empresa pode colher impressões com os consumidores, observar a aceitação do 
produto e desenvolver relacionamentos com o cliente, possibilitando a coleta de informações importantes para o PDP.

\subsection{Identificação do sucesso de novos produtos lançados após os diferentes níveis de envolvimento dos consumidores em PDP}

Nesta seção, apresentam-se os resultados obtidos a partir da análise da relação entre o sucesso dos novos produtos lançados no mercado e os diferentes níveis de envolvimento dos consumidores em PDP, ou seja, analisou-se se quanto maior o nível de envolvimento dos consumidores em PDP, maior o sucesso dos produtos lançados.

Com o objetivo de criar um modelo equacional capaz de prever o sucesso das empresas (variável dependente) através das variáveis independentes, que são os fatores PDP e Inovação e a variável nominal que classifica as empresas em vencedoras (0) e classificadas (1), nos prêmios FINEP e Nacional de Inovação, recorreu-se a técnica estatística de Regressão Linear com variável Dummy, conforme apresentados os resultados a seguir.

O modelo testado utiliza como variáveis preditoras os fatores Inovação, PDP e a variável dummy classificação, com o objetivo de gerar um modelo capaz de estimar a variável dependente, que é o fator Sucesso. A Tabela 1 mostra o valor do coeficiente de determinação, R2, que é igual a 0,015. Isso quer dizer que apenas $1,5 \%$ da variação da variável Sucesso é predita por este modelo, indicando que o mesmo não é preditivo, ou seja, a classificação da empresa (vencedora ou classificada nos prêmios FINEP e Nacional de Inovação), não foi capaz de indicar diferenças significativas sobre a dependência da variável sucesso em relação a inovação e PDP.

Tabela 1: Resumo do Modelo de Regressão Linear

\begin{tabular}{|c|c|c|c|c|}
\hline Modelo & $\mathrm{R}$ & $\mathrm{R}$ Square & Adjusted R Square & Std. Error of the Estimate \\
\hline 1 &, $301^{\mathrm{a}}$ &, 091 &, 015 &, 46811 \\
\hline \multicolumn{5}{|c|}{ a. Predictors: (Constant), Classificação, Inovação, PDP } \\
\hline \multicolumn{6}{|c|}{ b. Dependent Variable: Sucesso } \\
\hline
\end{tabular}

Fonte: Primária, 2012

A tabela 2, apresentada a seguir, mostra que o modelo estudado não é significativo, pois o valor-p=0,326, o que significa que os resultados coletados não conseguem classificar que as empresas vencedoras dos prêmios FINEP e Nacional de Inovação, utilizam mecanismos de interação com o consumidor de forma mais desenvolvida do que as empresas apenas classificadas nos prêmios citados, 
ou seja, não foi possível encontrar diferenças significativas entre os mecanismos de envolvimento do consumidor em PDP, utilizadas pelos dois grupos estudados.

Tabela 2: ANOVA da Regressão Linear

\begin{tabular}{|c|c|c|c|c|c|c|}
\hline \multicolumn{2}{|c|}{ Model } & Sum of Squares & DF & Mean Square & F & Sig. \\
\hline \multirow{3}{*}{1} & Regression &, 785 & 3 &, 262 & 1,194 &, $326 \mathrm{~b}$ \\
\cline { 2 - 7 } & Residual & 7,888 & 36 &, 219 & & \\
\cline { 2 - 7 } & Total & 8,673 & 39 & & & \\
\hline \multicolumn{7}{|c|}{ a. Dependent Variable: Sucesso } \\
\hline
\end{tabular}

Fonte: Primária, 2012

A tabela 3 apresenta os testes dos coeficientes das variáveis preditoras. Em outras palavras, apresenta a importância de cada variável na predição do Sucesso. Como pode-se observar na coluna "Sig.", nenhuma variável preditora foi significativa (todas com valores-p > 0,10). Inclusive a variável dummy (Classificação), que caso fosse significativa, mostraria a contribuição do fato da variável ser vencedora na previsão do sucesso da empresa.

Tabela 3: Testes dos Coeficientes de Regressão

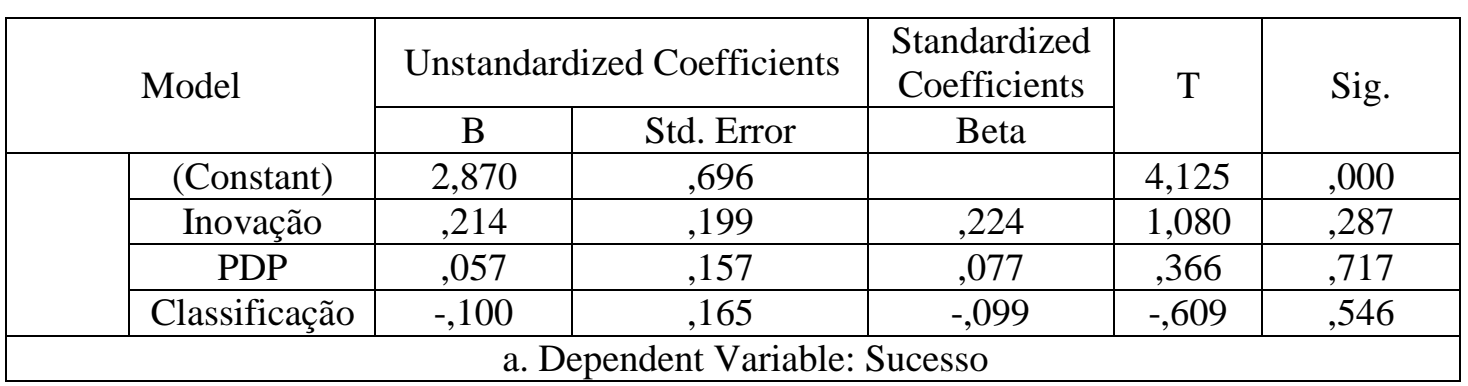

Fonte: Primária, 2012

Os resultados mostram que a variável dependente (sucesso), não sofre alteração de acordo com o comportamento das variáveis independentes (Inovação e PDP), ou seja, não foi possível determinar que uma ou outra variável tenha realmente influenciado os resultados dos prêmios pesquisados.

Este resultado destoa do foco dos prêmios estudados que deveriam premiar as empresas que se destacam em inovação, o que pode indicar que as empresas estudadas não apresentam diferenças significativas entre si, ou seja, empresas classificadas e vencedoras dos prêmios em estudo apresentam os mesmos comportamentos nas variáveis PDP e inovação em relação ao sucesso de seus produtos, deixando subjetivos os critérios de julgamento utilizados nestes prêmios. 


\subsection{Comparação entre PDP, Inovação e Sucesso entre Empresas Vencedoras e Classificadas nos Prêmios FINEP e Nacional de Inovação}

Nesta seção, utilizou-se para análise o teste não paramétrico Mann-Whitney, um teste de distribuição livre, ou seja, em que nenhuma suposição sobre a distribuição dos dados é necessária. Pode-se observar na Tabela 4 que tanto o teste Kolmogorov-Smirnov quanto o teste Shapiro-Wilk rejeitam a hipótese de normalidade dos dados, para todos os construtos, pois o valor-p é menor que 0,05 (coluna Sig. na Tabela 2). Apenas o teste Shapiro-Wilk, para variável Sucesso, não rejeita a hipótese de normalidade, ou seja, este teste mostra que os dados utilizados seguem uma distribuição normal.

Tabela 4: Teste de Normalidade dos Dados

\begin{tabular}{|c|c|c|c|c|c|c|}
\hline \multirow{2}{*}{} & \multicolumn{3}{|c|}{ Kolmogorov-Smirnova } & \multicolumn{3}{c|}{ Shapiro-Wilk } \\
\cline { 2 - 7 } & Statistic & DF & Sig. & Statistic & DF & Sig. \\
\hline Inovação &, 165 & 40 &, 007 &, 925 & 40 &, 011 \\
\hline PDP &, 269 & 40 &, 000 &, 753 & 40 &, 000 \\
\hline Sucesso &, 159 & 40 &, 012 &, 955 & 40 &, 111 \\
\hline \multicolumn{7}{|c|}{ a. Lilliefors Significance Correction } \\
\hline
\end{tabular}

Fonte: Primário, 2012

Após o teste de normalidade dos dados utilizou-se análise discriminante com o objetivo de identificar quais variáveis melhor diferenciam dois ou mais grupos. As variáveis Inovação, PDP e Sucesso, utilizadas neste estudo, foram agrupadas entre vencedoras e finalistas dos prêmios FINEP e Nacional de Inovação, a utilização destas variáveis foi realizada para se criar um índice ou função discriminante que represente de forma parcimoniosa as diferenças entre os grupos, classificar a priori novos indivíduos nos grupos.

O primeiro teste desta análise é o de igualdade de médias dos grupos, que visa identificar quais variáveis tem maior poder discriminador para os grupos em estudo. A variável com menor valor na estatística Wilk's Lambda é a mais discriminadora. Também é apresentado o teste F-ANOVA, que auxilia na interpretação e avaliação do teste anterior, apresentando o nível de significância de cada variável, que sendo inferior a 0,05 , indica diferença significativa entre os grupos naquela variável. Pelos testes a seguir pode-se verificar que nenhuma das variáveis apresenta poder discriminador, pois os valores-p foram todos maiores que 0,05 .

Revista de Administração e Inovação, São Paulo, v.12, n.1, p.174-200, jan./mar. 2015. 
Envolvimento do consumidor no processo de desenvolvimento de produtos como medida de sucesso: Um estudo com empresas participantes dos prêmios Finep e Nacional de Inovação

Tabela 5: Teste de igualdade das médias dos grupos

\begin{tabular}{|c|c|c|c|c|c|}
\hline & Wilks' Lambda & F & df1 & df2 & Sig. \\
\hline Inovação & 1,000 &, 009 & 1 & 38 &, 923 \\
\hline PDP &, 975 &, 967 & 1 & 38 &, 332 \\
\hline Sucesso &, 987 &, 502 & 1 & 38 &, 483 \\
\hline
\end{tabular}

Fonte: Primária, 2012

O teste na Tabela 6 verifica a significâncias da função discriminante. Pelo teste conclui-se que ela não é estatisticamente significativa, pois o p-value (Sig.) foi 0,628; maior que 0,05.

Tabela 6: Teste Wilks' Lambda

\begin{tabular}{|c|c|c|c|c|}
\hline Test of Function(s) & Wilks' Lambda & Chi-square & DF & Sig. \\
\hline 1 &, 953 & 1,741 & 3 &, 628 \\
\hline
\end{tabular}

Fonte: Primária, 2012

A Tabela 7, a seguir, apresenta os coeficientes padronizados das funções discriminantes. Normalmente as variáveis com maiores valores absolutos são as que mais contribuem para o poder discriminante de uma função.

Tabela 7: Coeficientes padronizados das funções discriminantes

\begin{tabular}{|c|c|}
\hline & Function \\
\cline { 2 - 2 } & 1 \\
\hline Inovação &,- 774 \\
\hline PDP & 1,111 \\
\hline Sucesso &, 487 \\
\hline
\end{tabular}

Fonte: Primária, 2012

A tabela a seguir mostra a classificação original da empresa e a classificação da mesma através da análise discriminante. Como apontavam os resultados anteriores, que mostraram que a função discriminante não foi significativa, o modelo não conseguiu classificar nenhum caso em empresa vencedora, colocando todas as empresas da amostra na condição de classificada. 
Tabela 8: Resultados da Classificação

\begin{tabular}{|c|c|c|c|c|c|}
\hline & & \multirow{2}{*}{ Classificação } & \multicolumn{2}{|c|}{ Predicted Group Membership } & \multirow{2}{*}{ Total } \\
\hline & & & Vencedora & classificada & \\
\hline \multirow{4}{*}{ Original } & \multirow{2}{*}{ Count } & Vencedora & 0 & 12 & 12 \\
\hline & & Classificada & 0 & 28 & 28 \\
\hline & \multirow{2}{*}{$\%$} & Vencedora & ,0 & 100,0 & 100,0 \\
\hline & & classificada & ,0 & 100,0 & 100,0 \\
\hline
\end{tabular}

Fonte: Primária, 2012

Os testes realizados não conseguiram determinar diferenças entre as empresas vencedoras e classificadas nos prêmios estudados, o que pode remeter a não confiabilidade nos resultados dos prêmios, pois não há diferenças significativas entre os grupos nas variáveis estudadas.

\subsubsection{Classificação da Empresa e Inovação; Classificação da Empresa e PDP; e Classificação da Empresa e Sucesso}

Nesta seção verificou-se através do teste Mann-Whitney se empresas vencedoras dos prêmios FINEP e Nacional de Inovação, apresentavam ou não, melhores índices de inovação, PDP e sucesso do que as demais empresas finalistas do prêmio.

Como resultado do teste classificação da empresa x Inovação, o valor do teste Mann-Whitney foi de 163,500 valor-p=0,901, ou seja, não encontrou-se diferença significativa entre os dois grupos de empresas.

Tabela 09: Teste de Mann-Whitney entre classificação da empresa e Inovação

\begin{tabular}{|c|c|}
\hline & Inovação \\
\hline Mann-Whitney U & 163,500 \\
\hline Wilcoxon W & 241,500 \\
\hline$Z$ &,- 134 \\
\hline Asymp. Sig. (2-tailed) &, 893 \\
\hline Exact Sig. [2*(1-tailed Sig.)] &, $896 \mathrm{~b}$ \\
\hline Exact Sig. (2-tailed) &, 901 \\
\hline Exact Sig. (1-tailed) &, 450 \\
\hline Point Probability &, 006 \\
\hline a. Grouping Variable: Classificação \\
\hline \multicolumn{2}{|c|}{ b. Not corrected for ties. } \\
\hline \multicolumn{2}{|c}{ Fonte: Primaria 2012 } \\
\hline
\end{tabular}

Fonte: Primária, 2012 
Envolvimento do consumidor no processo de desenvolvimento de produtos como medida de sucesso: Um estudo com empresas participantes dos prêmios Finep e Nacional de Inovação

Como resultado do teste classificação da empresa x PDP, o valor do teste Mann-Whitney foi de 139,500 valor-p=0,388, ou seja, não encontrou-se diferença significativa entre os dois grupos de empresas.

Tabela 10: Teste Mann-Whitney entre classificação da empresa e PDP

\begin{tabular}{|c|c|}
\hline & PDP \\
\hline Mann-Whitney U & 139,500 \\
\hline Wilcoxon W & 545,500 \\
\hline Z &,- 881 \\
\hline Asymp. Sig. (2-tailed) &, 378 \\
\hline Exact Sig. [2*(1-tailed Sig.)] &, $405 \mathrm{~b}$ \\
\hline Exact Sig. (2-tailed) &, 388 \\
\hline Exact Sig. (1-tailed) &, 193 \\
\hline Point Probability &, 007 \\
\hline \multicolumn{2}{|c|}{ a. Grouping Variable: Classificação } \\
\hline \multicolumn{2}{|c|}{ b. Not corrected for ties. } \\
\hline \multicolumn{2}{|c|}{ Fon Pritar } \\
\hline
\end{tabular}

Fonte: Primária, 2012

Como resultado do teste classificação da empresa x Sucesso, o valor do teste Mann-Whitney foi de 134,500 valor-p=0,322, ou seja, não encontrou-se diferença significativa entre os dois grupos de empresas.

Tabela 11: Teste Mann-Whitney entre classificação da empresa e Sucesso

\begin{tabular}{|c|c|}
\hline & Sucesso \\
\hline Mann-Whitney U & 134,500 \\
\hline Wilcoxon W & 540,500 \\
\hline Z & $-1,008$ \\
\hline Asymp. Sig. (2-tailed) &, 313 \\
\hline Exact Sig. [2*(1-tailed Sig.)] &, $328 \mathrm{~b}$ \\
\hline Exact Sig. (2-tailed) &, 322 \\
\hline Exact Sig. (1-tailed) &, 159 \\
\hline Point Probability &, 003 \\
\hline \multicolumn{2}{|c|}{ a. Grouping Variable: Classificação } \\
\hline \multicolumn{2}{|c|}{ b. Not corrected for ties. } \\
\hline
\end{tabular}

Fonte: Primária, 2012

\subsection{Discussão dos Resultados}

Neste item são discutidos os resultados desta pesquisa, as análises são feitas com o apoio da literatura e com base nos resultados obtidos, buscando favorecer os estudos sobre inovação e PDP, cocriação de valor e sucesso de novos produtos. 
A discussão acerca dos resultados encontrados sobre os níveis de envolvimento dos consumidores com as empresas estudadas baseia-se no entendimento de que os clientes não compram bens e serviços e sim itens que lhes retornam serviços que criam valor, ou seja, o marketing evoluiu de uma visão que focava no que as empresas pensavam para uma visão baseada no que os clientes necessitam (VARGO e LUSCH, 2004; ARNOULD e THOMPSON, 2005).

Utilizando-se os resultados da pesquisa, percebe-se que apesar de a literatura "apontar" o caminho para a satisfação e fidelização dos consumidores, as empresas não tem conseguido, em sua maioria, ou não tem se esforçado o suficiente, para utilizar todos os mecanismos disponíveis de envolvimento com os consumidores. $\mathrm{O}$ que se percebe é que grande parte das empresas acabam restritas aos tipos básicos de envolvimento, que lhes custam poucos investimentos, mas também não garantem todos os bons resultados possíveis.

Kaulio (1998) analisou as ferramentas de envolvimento com o consumidor, mais difundidas e utilizadas pelas empresas. Cada ferramenta foi analisada em duas dimensões: a intensidade de participação do cliente e em que etapas do PDP esta participação acontece. A pesquisa mostrou que tipos de envolvimento com o consumidor, com intensidades diferentes são usadas por empresas, dependendo do seu segmento e área de atuação. As empresas objeto desta pesquisa são dos mais variados segmentos, desde fabricação de aeronaves, passando por softwares, utilidades domésticas, setor cerâmico, entre outros, sendo que este fator pode ter contribuído para a não significância encontrada nos resultados da pesquisa, principalmente pelo fato de que algumas empresas da amostra enfrentam pouca concorrência no mercado, o que pode fazer com que não se empenhem suficientemente para envolver seus consumidores em PDP.

Os resultados obtidos nesta pesquisa, não corroboraram com a teoria, que diz que quanto maior o envolvimento do consumidor, maior o sucesso dos produtos lançados, pois as análises não demonstraram diferenças significativas entre os níveis de envolvimento de empresas vencedoras e classificadas nos prêmios utilizados como referência para a pesquisa.

Percebe-se porem, que as empresas do universo da pesquisa, para concorrer aos prêmios, podem ter criado produtos ou projetos especificamente para este fim, que talvez apresentassem características inovadoras, mas que não significassem bens de interesse do consumidor, outra possibilidade é que as características de algumas empresas, de terem como clientes outras empresas, podem fazer com que influências externas alheias a esta pesquisa acabem interferindo nos resultados dos prêmios, uma vez que as empresas não são escolhidas aleatoriamente e sim mediante inscrição no prêmio, as empresas participantes dos prêmios e os prêmios, podem ainda ter caráter de promoção da 
inovação e não de buscar a inovação, ou seja, vencer os prêmios pode não indicar que a empresa seja inovadora, apenas que soube desenvolver um projeto ou produto inovador.

Clark e Wheelwright (1993) afirmam que uma das causas ligadas ao não sucesso no PDP pode ser a não articulação entre projetos de novos produtos e a estratégia da empresa. O que indica que todas as "frentes" da organização devem estar voltadas para a mesma direção, quando se objetiva o sucesso no lançamento de novos produtos. No caso das empresas estudadas, se o perfil inovador realmente refletir-se apenas em alguns projetos e não na cultura organizacional, é bem possível que essas organizações realmente não se diferenciem das demais do mercado, no sucesso de lançamento de novos produtos.

Os resultados encontrados nesta pesquisa indicam que ao se fazer a comparação das variáveis PDP e inovação entre as empresas vencedoras e classificadas nos prêmios FINEP e Nacional de Inovação, não foi possível constatar-se diferenças significativas. Para Hart (1993) há uma forte relação entre as empresas com tendência à inovação e o sucesso no mercado.

Entende-se que a teoria sobre sucesso no mercado para empresas inovadoras tem se mostrado confirmatória em vários estudos, porem no caso deste estudo, os indicadores mostram que os prêmios estudados não verificam a fundo o desenvolvimento da inovação nas empresas participantes, apenas no produto ou projeto inscrito no prêmio, que pode ter sido desenvolvido apenas para a ocasião do prêmio.

\section{CONCLUSÃO}

Este trabalho procurou responder quais os níveis de envolvimento dos consumidores em processos de PDP, como medida de sucesso no lançamento de novos produtos. O objetivo foi de analisar os níveis de envolvimento dos consumidores em processos de PDP, como medida de sucesso no lançamento de novos produtos. Este objetivo foi atingido conforme a pesquisa foi realizada com base nos levantamentos teóricos, analisando-se a participação de consumidores em PDP, a inovação e sua influência no sucesso de novos produtos. A utilização do questionário em parte adaptado de Santos e Brasil (2010) e em parte de Damanpour (1991) permitiu a concretização deste objetivo, por meio da análise das respostas dos responsáveis pelo PDP das empresas participantes da amostra.

A pesquisa iniciou-se em fontes de referências bibliográficas de relevância na academia, utilizando-se de artigos nacionais e estrangeiros, para que conhecesse conceitos como Estratégia de 
PDP, Inovação, Comportamento do Consumidor, Cocriação de Valor, Medidas de Sucesso e a relação entre as variáveis utilizadas.

Em relação à contribuição teórica, pode-se perceber que os estudos de Prahalad e Ramaswany (2004); Slater e Narver (1995); Jugend et al, 2006; Brown e Eisenhardt (1995), que dizem que as empresas precisam cocriar valor para obter vantagem de seus concorrentes e assim, obter sucesso no lançamento de novos produtos, foram confirmados por este trabalho.

$\mathrm{Na}$ fundamentação teórica foram encontrados estudos com implicações gerenciais como Von Hippel (1986); Lilien et al (2002); Alam (2002); Nambisan (2002), que corroboram com a defesa das vantagens do envolvimento de consumidores realizada por Prahalad e Ramaswamy (2004) e Vargo e Lusch (2004), no entanto, ainda no início do estudo foi possível verificar que existem lacunas entre a conceituação do tema e a realidade das empresas estudadas e apesar de esta pesquisa ter-se baseado em várias publicações nacionais e internacionais, não encontrou-se nenhuma pesquisa integrando a relação entre PDP, inovação e sucesso no lançamento de novos produtos, e apenas um estudo sobre os mecanismos de interação com o consumidor utilizados por empresas vencedoras de Prêmios de Inovação.

A importância deste trabalho dá-se por uma abordagem diferente entre os critérios que envolvem PDP, inovação e sucesso de forma conjunta, contribuindo com estudos sobre fatores que influenciam o sucesso de novos produtos e a importância da cocriação em PDP, principalmente pelas facilidades de entradas em novos mercados e aumento exponencial da concorrência.

Percebe-se também a importância deste estudo, ao discutir a verdadeira finalidade e a subjetividade dos prêmios e dos critérios dos prêmios de inovação no Brasil, sendo assim, entende-se que é necessário que estes prêmios atuem em frentes mais consistentes do que produtos ou projetos inscrito, como por exemplo, a análise de um mix maior de produtos da empresa em questão, seria importante também que se estabelecessem critérios mais claros sobre o julgamento dos prêmios, permitindo que não ocorram dúvidas sobre a fidedignidade dos prêmios.

\subsection{Limitações da Pesquisa}

O estudo realizado apresentou limitações importantes quanto a sua população e amostra. A população definida para este estudo, empresas vencedoras e classificadas nos prêmios FINEP e Nacional de Inovação, entre os anos 2008 e 2011, mostrou relativamente pequena, pois apesar do grande número de empresas premiadas em cada edição dos prêmios, em muitos casos a mesma empresa ganhou o prêmio por mais de um ano, ou em mais de uma categoria e às muitas vezes a

Revista de Administração e Inovação, São Paulo, v.12, n.1, p.174-200, jan./mar. 2015. 
empresa vencedora de um prêmio foi também a vencedora do outro prêmio estudado, resultando em uma população de 72 empresas.

Outro fator limitante é que o fato de os prêmios premiarem em alguns casos ações das empresas e em outros produtos desenvolvidos pelas empresas, o que pode fazer com que algumas organizações tenham preparado produtos "especiais" para concorrer aos prêmios, aplicando neles mais inovação e utilizando mais cocriação do que de costume, fazendo com que aquele produto não reflita a realidade da empresa.

Outro importante fator limitador é a falta de definições claras sobre os julgamentos dos prêmios, se há ou não critérios subjetivos no momento da premiação, como por exemplo, se as empresas participantes apoiam em forma de patrocínio a realização dos prêmios, se algum dos avaliadores possui algum tipo de vínculo profissional ou pessoal com alguma empresa.

Outra limitação se deve ao fato de que as empresas devem se inscrever para participar dos prêmios, o que sugere que há possibilidade de empresas com PDP extremamente desenvolvido, com sucesso no lançamento de seus produtos, mas que não tenham se inscrito no prêmio. Entende-se que as limitações encontradas podem ter interferido nos resultados da pesquisa, fazendo com que as não significâncias de resultado ocorressem.

\subsection{Implicações Gerenciais}

Diante dos resultados obtidos com esta pesquisa e do levantamento teórico realizado durante este estudo, sugere-se que as empresas interessadas em participar e vencer os prêmios, FINEP e Nacional de Inovação devem participar mais ativamente junto as agências idealizadoras destes prêmios e investir de forma que ganhem maior visibilidade junto a esses órgãos, pois estar presente em prêmios de inovação pode vincular positivamente a imagem da organização com posturas inovadoras.

O fato dos prêmios FINEP e Nacional de Inovação avaliarem apenas projetos de empresas que se inscreveram para eles, podem fazer com que organizações que ficaram de fora dos prêmios, mas possuem métodos de interação mais desenvolvidos e maior inovatividade, não sejam reconhecidas como tal.

Apesar deste estudo não ter encontrado relação entre inovação, PDP e sucesso de novos produtos dentro da amostra analisada, percebeu-se que as organizações perdem oportunidades de sucesso por não investir em desenvolvimento no setor de PDP, o que é amplamente confirmado pela literatura (OLIVEIRA et al, 2006; SLACK et al., 2002; BROWN e EISENHARDT, 1995).

Revista de Administração e Inovação, São Paulo, v.12, n.1, p.174-200, jan./mar. 2015. 
Entende-se também, através da literatura levantada que o investimento em melhorar o PDP das organizações trás resultados positivos para a mesma, sendo na diminuição de custos de pesquisas ou do tempo de lançamento dos produtos, ou seja, mesmo que os investimentos em PDP não resultem em prêmios, por fatores alheios aos critérios dos prêmios, os resultados junto ao consumidor se mostram satisfatórios, e o reconhecimento pode aparecer em números de vendas.

Com base na subjetividade encontrada para a análise dos produtos e/ou empresa concorrente aos prêmios FINEP e Nacional de Inovação entende-se que os organizadores do prêmio poderiam considerar o ambiente de inovação da organização concorrente, mesmo em categorias onde a avaliação do prêmio se refere a produtos, afinal, pode-se estar classificando uma empresa como inovadora, por ter ganhado o prêmio, baseado em critérios errôneos.

Outro fator a ser observado é a composição das comissões julgadoras, lembrando que os dois prêmios têm como responsáveis agências públicas. Segundo Finep (2013), as comissões regionais serão formadas por renomados profissionais das áreas de ciência, tecnologia e comunicação e por empresários e representantes de entidades do setor; a comissão nacional será composta por um presidente e especialistas das áreas científicas e tecnológicas de diferentes estados; o júri é soberano e sobre o resultado não caberá recurso.

Sugere-se aos organizadores dos prêmios citados, avaliação "às cegas", ou seja, que o avaliador só tenha acesso aos dados avaliados nos prêmios, e em hipótese nenhuma conheçam as características nominais dos projetos e organizações que estão avaliando. Esse tipo de procedimento não é citado em nenhum dos regulamentos dos prêmios estudados, o que pode gerar desconforto para os avaliadores ou ainda favorecimento para uma ou outra organização por relacionamentos alheios ao prêmio entre empresa e avaliador.

\subsection{Sugestões para Trabalhos Futuros}

A partir da realização deste trabalho, foi possível verificar que ainda há muito a avançar na compreensão sobre o envolvimento de consumidores, ou ainda sobre a cocriação de valor. O tema ainda considerado emergente necessita ser aprofundado e testado sob diferentes dimensões. Esse trabalho se apoiou na descoberta de quais os níveis de envolvimento dos consumidores em processos de PDP, como medida de sucesso no lançamento de novos produtos?, no entanto, há lacunas que ainda precisam ser preenchidas para compreensão total sobre o tema. Sendo assim, apresentam-se as seguintes sugestões:

Revista de Administração e Inovação, São Paulo, v.12, n.1, p.174-200, jan./mar. 2015. 
- Realizar trabalhos utilizando todo o histórico de empresas vencedoras e classificadas nos prêmios em estudo, gerando uma maior população para estudo.

- Analisar os dados secundários de empresas de capital aberto, sendo possível a análise de informações que ficam obrigatoriamente disponíveis em seus sites;

- Analisar os critérios subjetivos utilizados pelos prêmios, em estudo nesta pesquisa, objetivando dar uma visão mais realista sobre o funcionamento dos prêmios e sua idoneidade;

- Realizar um trabalho qualitativo com as empresas selecionadas, objetivando conseguir mais elementos para a análise do ambiente de inovação e cocriação das empresas.

- Avaliar grupos de outros prêmios ligados a área de inovação e cocriação.

- Realizar de estudos de cocriação em empresas de prestação de serviços.

- Realizar uma pesquisa qualitativa com os organizadores dos prêmios para verificar quais os critérios utilizados nos prêmios, e se coincidem a teoria sobre o tema.

\section{REFERÊNCIAS}

ALAM, I. An exploratory investigation of user involvement in new service development. Journal of the Academy of Marketing Science, v. 30, n. 3, p. 250-261, 2002.

ARNOULD, E. J., ; THOMPSON, C. J. (2005). Consumer culture theory (CCT): Twenty years of research. Journal of Consumer Research, 31, 868-882, (March).

BRANDALISE, L.T.; LEZANA, A.G.R.; ROJO, C.A. O comportamento do consumidor e sua percepção da variável ambiental: Um modelo de suporte à gestão empresarial. Revista TECAP Número 03 - Ano 3 - Volume 3 - 2009 annual

BROWN, S. L.; EISENHARDT, K.M. Product Development: Past Research. Present Findings, and Future Directions. Academy of Management Review. v. 20, p. 343-378, 1995.

CHANDLER, J.D. e VARGO, S.L. Contextualization and Value-in-context: How Context Frames Exchange. Marketing Theory. v. 11, n. 1, p. 35-49, 2011.

CHATHOTH, P. UNGSON, B. ALTINAY, L. HARRINGTON, R. OKUMUS, F., \& CHAN, E. Reorienting from Co-production to Co-creation: Challenges and Constraints in the Hotel Service Context. International Journal of Hospitality Management, 32 (1), 11-20, 2013.

Revista de Administração e Inovação, São Paulo, v.12, n.1, p.174-200, jan./mar. 2015. 
CLARK, Kim, WHEELRIGHT, Steven. Managing New Product and Process Development. Free Press, 1993.

DAMANPOUR, F. Organizational Innovation: A Meta- analysis of Effects of Determinants and Moderators, Academy of Management Journal, v.34, n.3, p. 555 - 590, 1991.

DOGSON, M.; GANN, D.; SALTER, A. The role of technology in the shift towards open innovation: The case of Procter \& Gamble. R\&D Management, v. 36, n.3, p. 333-346, 2006.

EDVARDSSON, B.; TRONVOLL, B.; GRUBER; T. Expanding understanding of service exchange and value co-creation: a social construction approach. Journal of the Academy of Marketing Science, Volume 39, Issue 2, pp 327-339, 2010.

FINOTTI, M. A. Contribuições ao estudo dos estilos de vida: comportamento de compra e uso de crédito. Tese (Doutorado acadêmico em Administração). Universidade de São Paulo, São Paulo, 2009

GARCIA, F. J. Um Estudo sobre as Formas de Inovação e os Critérios de Avaliação dos Prêmios de Inovação. Dissertação (Mestrado) - Universidade de Caxias do Sul, Programa de Pós-Graduação em Administração, 141 f, 2008.

GOMES, E.S. e BARBOSA, E.F. A Técnica de Grupos Focais para Obtenção de Dados Qualitativos. Instituto de Pesquisa e Inovações Educacionais. 1999.

GRIFFIN, A. A PDMA Research on New Product Development Practices: Updating Trends and Benchmarking Best Practices. J. Prod. Innov. Manag. v. 14, p. 429-458, 1997.

HART, S. Dimensions of success in new product development: an exploratory investigation. Journal of Marketing Management. v. 9, p. 23-41, 1993.

HUGHES S, TIPPETT D, THOMAS W. Measuring Project Success in the Construction Industry. Engineering Management Journal. v. 16, n. 3. pág. 31-37. September 2004

Instituto Inovação. Prêmios da Inovação: Ferramentas para Promoção da Cultura da Inovação Tecnológica nas Empresas. 15p. 2005.

JUGEND, D. Desenvolvimento de Produto em Pequenas e Médias Empresas de Base Tecnológica: Práticas de Gestão no Setor de Automação de Controle de Processos. São

Carlos, 2006. 125p. Dissertação (Mestrado em Engenharia Product: Management \& Development 125 de Produção) - Universidade Federal de São Carlos, Departamento de Engenharia de Produção.

Revista de Administração e Inovação, São Paulo, v.12, n.1, p.174-200, jan./mar. 2015. 
KAULIO, M.A. Customer, consumer and user involvement in product development: a framework and a review of selected methods. Total Quality Management, v. 9, n. 1, 1998.

LILIEN, G.L. et al. Performance Assessment of the Lead User Idea-Generation Process for New Product Development. Management Science, v. 48, n. 8, Ago. 2002.

LUSCH, R. F. Marketing's envolving identify: defining our future. American Marketing, v. 26, n. 2, 2007.

MALHOTRA. Naresh K. Pesquisa de Marketing: uma orientação aplicada. São Paulo. Bookman, 2006.

NAMBISAN, S. Designing virtual customer environments for new product development. Academy of Management Review, v 27, p.392-413, 2002.

NAMBISAN, S; BARON, R. A. Interactions in virtual customer environments: implications for product support and customer relationship management. Journal of Interactive Marketing, v. 21, n. 2, 2007.

OLIVEIRA, G.T. de; MAIA, J.L.; MARTINS, R.A. Estratégia de produção e desenvolvimento de produto em uma empresa do setor de cosméticos. SISTEMAS \& GESTÃ O, v. 1, n. 1, p. 58-74, jan/abr de 2006.

PRAHALAD, C. K; RAMASWAMY, V. Co-creation experiences: the next practice in value creation. Journal of Interactive Marketing, v. 18, n. 3, 2004.

ROZENFELD, H. et al. Gestão de desenvolvimento de produtos: uma referência para a melhoria do processo. São Paulo: Saraiva, 2006.

SANTOS, Carolina Rosado Dos; BRASIL, Vinícius Sittoni. Envolvimento do Consumidor em Processos de Desenvolvimento de Produtos: Um Estudo Qualitativo Junto a Empresas de Bens de Consumo. Revista de Administração de Empresas: RAE, São Paulo, v. 50, n. 3, p.300-311, jul./set. 2010.

SAWHNEY, M., VERONA, G., PRANDELLI, E. Collaboration to Create: the Internet as a Platform for Customer Engagement in Product Innovation. Journal of Interactive Marketing. vol. 19, issue 4, Autumn 2005, pp. $4-17$ 
SBRAGIA, Roberto e ANDREASSI, Tales. Relações entre Indicadores de P\&D e de Resultado Empresarial. Revista de Administração - USP. v. 37, n. 1. jan/mar 2002.

SHOCKER, A.D., SRINIVASAN, V. Multiattribute approaches for product concept evaluation and generation: A critical review. J. Marketing Res. v. 16 p. 159-180, 1979.

SKINNER, W. Manufacturing - missing link in corporate strategy. Harvard Business Review. Boston, Vol. 47, N. 3, p.136-145, May-June. 1969.

SLACK, N. CHAMBERS, S. JOHNSTON, R. Administração da Produção. $2^{a}$ ed. São Paulo: Atlas, 2002.

SLATER, S. F; NARVER, J.C. Market orientation and the learning organization. Journal of Marketing, v. 59, n. 3, 1995.

VARGO, S. L; LUSCH, R. F. Evolving to a new dominant logic for marketing. Journal of Marketing, v. 68 , n. 1, p. 1-17, 2004.

VENKATRAMAN, N., RAMANUJAM, V. Measurement of Business Performance in Strategy Research: A Comparison of Approaches. Academy Of Management Review, v. 11, n. 4 pág. 801-814. 1986.

VON HIPPEL, E. Lead Users: A source of novel products concepts. Management Science, v. 32, p. 791-805, 1986.

VON HIPPEL, E. The Sources of Innovation. New York: Oxford University Press, 1988. 


\begin{abstract}
This study aimed to analyze the levels of consumer involvement in processes of PDP (Product Development Process) as a measure of success in launching new products, besides discusses, from the results that were found, if the innovation awards in Brazil are really to reward products and/ or innovative projects, or are just for innovation promotion. To achieve the objectives of this work, we chose a quantitative and descriptive approach. Data collection was conducted through electronic questionnaire sent via email to the managers of the nominated and winners companies in the National Innovation and FINEP awards, between 2008 and 2011. For the multivariate statistical analysis, we used the nonparametric Mann-Whitney test, discriminant analysis and linear regression with dummy variable, besides the statistical analyzes using univariate analysis of variance ANOVA. The data analysis was performed using SPSS (Statistical Package for the Social Sciences) software. The results showed that $100 \%$ of the researched firms have some kind of mechanism to engage with consumers. It was also found that the least used interaction tool with the consumer is the virtual one.
\end{abstract}

Keywords: Innovation awards; The consumer involvement; PDP.

Data do recebimento do artigo: 05/04/2014

Data do aceite de publicação: 05/01/2015

Revista de Administração e Inovação, São Paulo, v.12, n.1, p.174-200, jan./mar. 2015. 